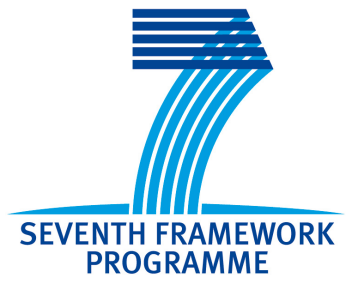

Project no. 248992

Project acronym: NEUNEU

Project title: Artificial Wet Neuronal Networks from Compartmentalised Excitable Chemical Media

Small or medium-scale focused research project (STREP)

Deliverable 2.5 - Report or publication on computation with three and more units

Duration: 36 months

Project coordinator name:

Dr. Peter Dittrich

Project coordinator organisation name: Friedrich Schiller University Jena 


\section{Information coding with frequency of oscillations in Belousov-Zhabotinsky encapsulated disks}

J. Gorecki ${ }^{a, b, *}$, J. N. Gorecka ${ }^{c}$ and Andrew Adamatzky ${ }^{d}$ ${ }^{a}$ Institute of Physical Chemistry, Polish Academy of Sciences, Kasprzaka 44/52, 01-224 Warsaw, Poland.

${ }^{b}$ Faculty of Mathematics and Natural Sciences, Cardinal Stefan Wyszynski University

Dewajtis 5, Warsaw, Poland

${ }^{c}$ Institute of Physics, Polish Academy of Sciences,

Al. Lotnikow 36/42, 02-668 Warsaw, Poland ${ }^{d}$ Unconventional Computing Centre and Department of Computer Science, University of the West of England, Bristol, England October 27, 2013

\footnotetext{
Abstract

Information processing with an excitable chemical medium, like
} 
Belousov-Zhabotinsky (BZ) reaction, is typically based on information coding in a presence or absence of excitation pulses. Here we offer an alternative approach. A medium represents the logical TRUE state if the frequency of oscillations at a selected domain exceeds a specified threshold; otherwise, the medium represents the logical FALSE state. We consider a medium composed of disks encapsulating an oscillatory mixture of reagents, as related to our recent experiments with lipid coated BZ droplets. We demonstrate that by using special geometrical arrangements of disks containing the oscillatory medium one can perform logical operations on variables coded in oscillation frequency. Realizations of a chemical signal diode and a memory ring with oscillatory disks are also discussed.

\section{Introduction}

The unconventional computing $[1,2,3,4]$ is a field of research dedicated to chemistry, physics or biology inspired computational strategies, structures, and substrates. In contrast to the conventional computer technology based on semiconductors and the von Neumann concept of computer architecture [5] the unconventional computing interprets the natural time evolution of a medium as a series of information processing operations. The time clock sequencing performed operations is hidden in speeds of considered phenomena and in interactions between different parts of the medium. Different types of media can be used for unconventional computation. Information processing operations executed by a chemical medium seem especially interesting, because the activity of nervous systems including that of our brain, are based 
on chemical reactions. An excitable medium is considered in the majority of studies on chemical information processing $[6,7]$. There are obvious qualitative analogies between the behaviour of a chemical excitable medium and of a nerve cell. If a perturbation of an excitable medium does not exceed the threshold value than the system remains at its stationary state and shows no activity that can be interpreted as information processing operations. Perturbations, that are sufficiently strong can generate excitations of the medium observed as high concentrations of selected reagents. If a perturbation is local and diffusion of reagents is allowed, than pulses of excitation formed by peaks of concentrations can propagate through the medium. In chemical information processing media, as well as in biological systems, information can be coded in the presence of excitations or in trains of excitation pulses.

The binary representation is the simplest information coding that can be implemented in a chemical medium. A high concentration of the reagent responsible for excitation observed at a given point of space and at a given time represents the logical TRUE state. A concentration below the assumed threshold is interpreted as the logical FALSE. This binary coding can be used if the medium is excitable or when the period of oscillations is longer than the time required for an excitation to propagate over a distance comparable with the system size. Such coding has been used in the majority of publications on information processing with excitable Belousov-Zhabotinsky (BZ) reaction $[6,7,8]$. Within such interpretation a propagating excitation pulse represents a bit of information traveling in space. Information is processed in loci of space, where pulses interact. As a consequence, in information processing applications, the geometrical structure of medium is equally important 
as the kinetics of reactions involved $[7,6]$. Complex information processing operations can be performed even for relatively simple reaction kinetics if the geometrical distribution of regions with different chemical properties is carefully prepared. By selecting a clever geometrical distribution of regions with high or low excitability level one can force interactions that lead to the required outcome like unidirectional propagation of signals (a chemical signal diode) [9] or a band pass signal filter [10]. An important benchmark for the usefulness of unconventional computing medium is its ability to reproduce the logic gates $[8,6]$. Logic gates are the physical embodiment of Boolean logic operations that form the foundation for digital information processing. Circuits of logic gates can be connected to create machines capable of performing universal computation [11]. If we show that a functionally complete set of logic gates can be constructed in a medium then the medium can be seen as a universal computer.

Studies on logic operations executed by excitation pulses have a long history. Toth and Showalter described logic gates with BZ waves traveling along thin capillary tubes [12]. Steinbock and collaborators implemented logic gates by 'printing' a catalyst of the BZ reaction onto a facilitating medium [13]. In simulation studies [8, 14] the construction of logic gates relies upon geometric patterns of non-excitable boundaries imposed on an excitable field. Adamatzky presented yet alternative logic gate designs by combining the principles of collision-based computing on an precipitating chemical substrate $[15,16]$.

In the series of recent papers Adamatzky and collaborators $[17,18,19,20$, 21] considered the logic gates as well as some selected arithmetic operations 
executed by a system of sub-excitable disks. Their numerical simulations were based on the Oregonator model for a photosensitive BZ reaction [22]. The simulation results were supported by experiments with Ru-catalised BZ reaction. In the experiments a membrane with immobilized catalyst was used as a nonlinear medium. The membrane was placed inside the solution of the other reagents of BZ-reaction. In such system the reaction proceeds on membrane surface. The areas of low-intensity illumination formed a subexcitable interiors of disks. The disks were separated by regions of highintensity illumination where excitation pulses decayed rapidly. The authors speculated that the medium composed of disks is qualitatively similar to a set of droplets containing the solution of BZ reaction separated by nonoscillatory organic phase [23].

The recent experiments performed within the NEUNEU project [24] have shown that it is relatively easy to make a structure composed of interacting droplets containing oscillatory BZ. On the other hand, it is seems difficult to prepare a similar structure with droplets containing an excitable medium and to control its local excitations. These experimental facts encouraged us to present here an alternative method of Boolean information coding that is based on oscillation frequency measured at selected points of the medium, like centers of individual droplets. We demonstrate that the ideas and realisations of information processing devices described in [20] for 2D disks containing sub-excitable medium and information coded in the presence of excitation pulses can be directly translated into the oscillatory medium with frequency based information coding. We discuss the construction of two basic logic gates - OR and NOT - that allow us to build all other gates. Moreover, we 
show that both a single-bit memory cell and a signal diode can be constructed using a few disks. Our results are based on simulations performed for exactly the same Oregonator model of a photosensitive BZ medium that has been used in $[17,18,19]$. A good qualitative agreement between simulations and experiments was demonstrated in those papers. Therefore, we expect that the presented disk-based constructions of gates, a signal diode and a memory cell can be also easily verified in experiments with a photosensitive BZ reaction and the Ru-catalyst immobilised on a membrane. On the other hand, simple, realistic models of kinetics of BZ droplets [25] are formally similar to the Oregonator model, so we think that a structure of oscillating lipid covered droplets that performs a given information processing function should be similar to the structure of disks presented in this paper.

\section{The information processing structures of oscillating disks}

\subsection{The basic properties of the model}

The idea of frequency based information coding comes from the observation that in a typical oscillatory medium the region with the highest oscillation frequency becomes a pacemaker that forces the neighboring areas to oscillate with the same frequency. The frequency of BZ oscillations can be controlled by many factors. In experiments with lipid covered BZ droplets frequency of oscillations depends on the initial concentrations of reagents and experimental conditions including temperature and illumination intensity. Here in numerical simulations based on a model of a photosensitive BZ reaction we 
fix the oscillation frequency by selecting the value of illumination level in a term that describes the influence of light on reaction kinetics. In our simulations we use exactly the same two-variable version of the Oregonator model adapted for photosensitive Ru-catalyzed medium as in [19]. Its equations read:

$$
\begin{gathered}
\frac{\partial u}{\partial t}=\frac{1}{\epsilon} \cdot\left[u-u^{2}-(f \cdot v+\phi) \frac{u-q}{u+q}\right]+D_{u} \nabla^{2} u \\
\frac{\partial v}{\partial t}=u-v+D_{v} \nabla^{2} u
\end{gathered}
$$

In these equations $u$ and $v$ denote the dimensionless concentrations of the bromous acid $\mathrm{HBrO}_{2}$, that is the reaction activator and of the oxidised form of the catalyst $R u(b p y)_{3}^{3+}$ that acts as its inhibitor.

In Eq.(1) $\phi$ is the rate of bromide production and it is proportional to the applied light intensity. Bromide $\mathrm{Br}^{-}$is an inhibitor of BZ-reaction and the Ru-catalysed BZ reaction can be controlled by light intensity. Experimental conditions can be set such that at low illuminations the medium oscillates, for larger light intensity it becomes excitable and for yet larger illumination it is sub-excitable. When exposes to a high-intensity of light the medium exhibits no nonlinear behaviour because it has a strongly attractive stable state.

For simulations reported below we selected the same values of model parameters as used in [19]. The ratio of time scales for the evolution of $u$ and $v$ variables $\epsilon=0.022$. The stoichiometric coefficient $f=1.4$ and $q=0.0002$. Like in [19] we assume that the mobility of catalyst can be neglected if compared to the activator. Thus the diffusion coefficients $D_{u}$ and 
$D_{v}$ of $u$ and $v$ were set to unity and zero respectively. To get numerical results presented below we solved Eqs.(1,2) using 4-th order Runge-Kutta algorithm with $d t=2 \cdot 10^{-5}$. One- and two- dimensional evolution was studied on a square grid with spatial steps $\Delta_{x}=\Delta_{y} \in[0.04,0.05]$. The exact value of the space grid was separately selected for each particular problem.

Fig. 1 illustrates the period of oscillations as a function of $\phi$ calculated using Eqs. $(1,2)$ with the considered parameter values. The range of $\phi$ where oscillations appear is very limited and oscillations are dumped for $\phi \geq 0.000477$. For the following analysis of of frequency coded information processing by structures of BZ-disks we considered two arbitrary selected illumination levels $\phi_{1}=0.0002$ and $\phi_{2}=0.00047$. The periods of homogeneous oscillations at these illuminations are $T_{1}=7.26554$ and $T_{2}=8.63774$ respectively and we use them as the inputs of binary, frequency coded information. These periods are visibly different, so we can introduce the following information coding. Let us select the threshold period $T_{c}=8.1$. We will assume that a medium oscillating with period shorter than $T_{c}$ represents the logical TRUE state. If the oscillation period is longer than $T_{c}$ than such oscillations represents the logical FALSE state. Of course, one can select another value of $T_{c}$, provided it is between $T_{1}$ and $T_{2}$ so the considered disk structures with illumination levels $\phi_{1}$ and $\phi_{2}$ can represent the both states of binary logic.

Experiments with lipid covered BZ droplets have shown that high frequency oscillations expand between droplets like excitation spikes. Therefore, we can expect that structures of disks for processing information coded in frequency of local oscillations are similar to those that process information coded in the presence of excitation pulses $[17,18,19,20,21]$. 

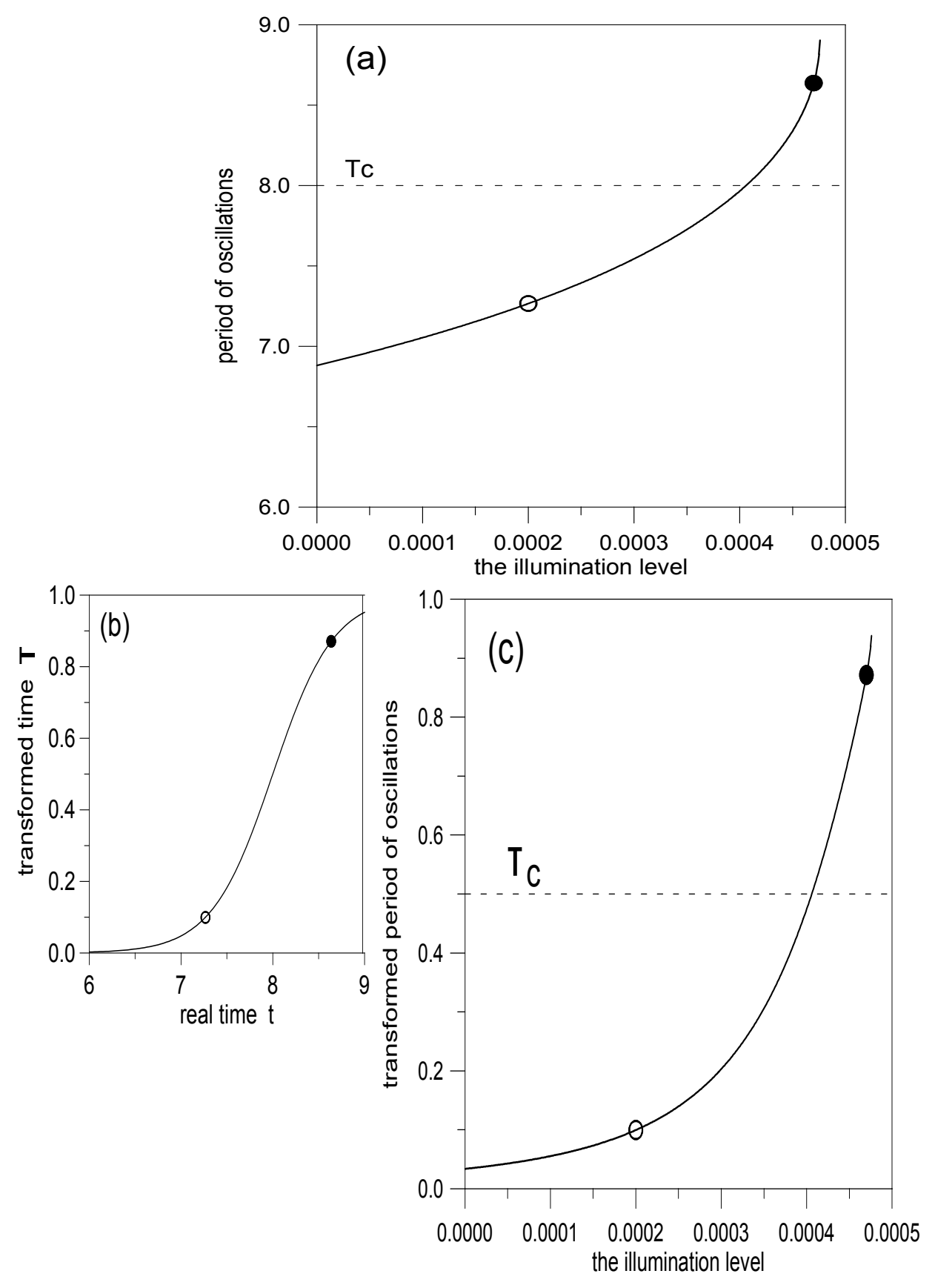

Figure 1: (a) The period of homogeneous oscillations as a function of illumination level $\phi$ calculated for the model parameters used in the paper. White and black circles mark periods of oscillations corresponding to the TRUE and the FALSE states. (b) The transformation of time introduced to enhance time differences around selected $T_{c}$. (c) The transformed period of homogeneous oscillations as a function of illumination level. 

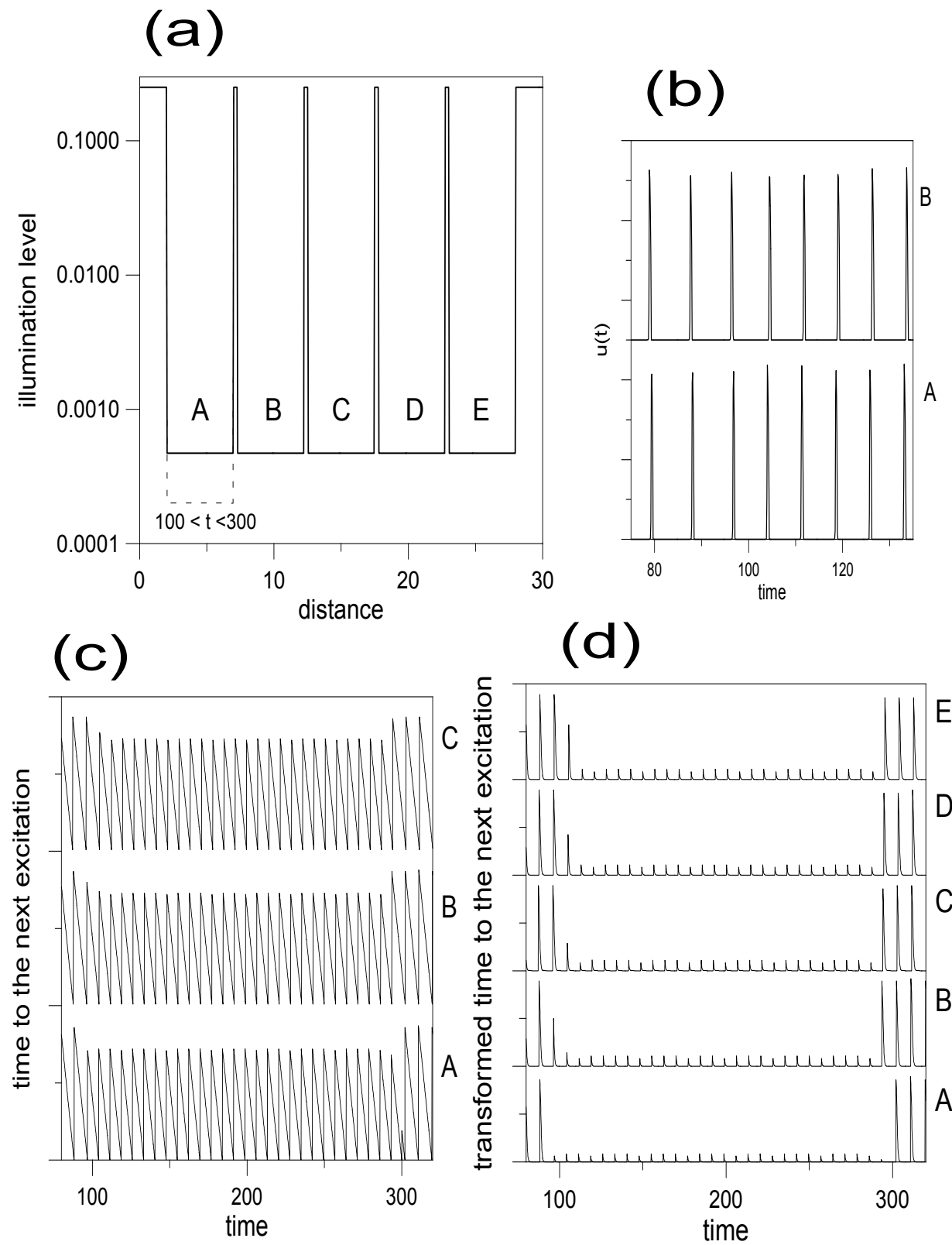

(d)

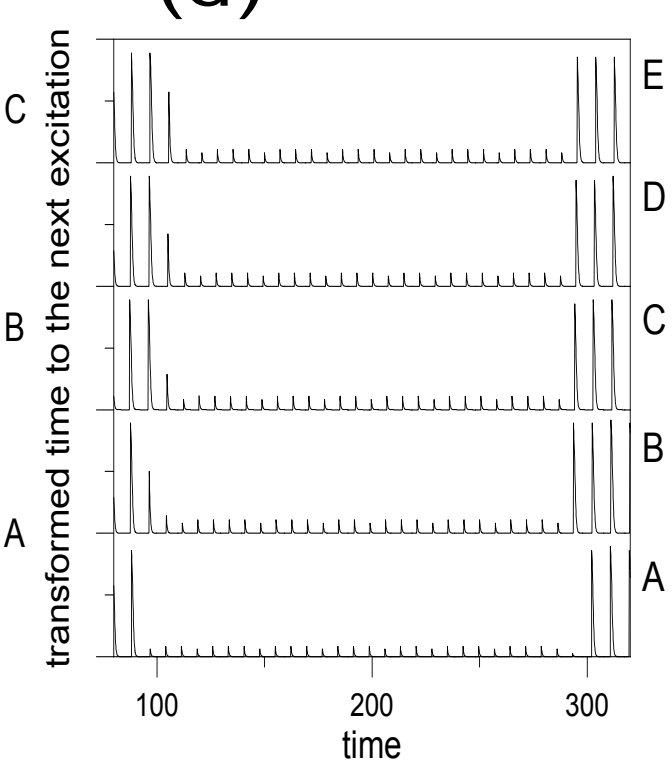

Figure 2: Local frequency of oscillations in one-dimensional medium with time dependent, inhomogeneous illumination. (a) The time dependent illumination of the medium. (b) The time evolution of activator concentrations in the centres of segments A and B. (c) $D(t)$ calculated at segment centres as a function of time. (d) $\tau(D(t))$ at the centres of all disks as a function of time. 
In order to demonstrate that locally forced high frequency oscillations in a medium oscillating with a low frequency behave qualitatively similar to pulses in an excitable medium let us consider one-dimensional system with non-homogeneous illumination. The system is composed of five dark segments (labeled A, B, C, D and E in Fig. 2a) that are 100 grid points wide $\left(\Delta_{x}=0.05\right)$. The segments are located on a strongly illuminated background ( illumination $\phi_{0}=0.25$ ). We assume that the activator $u$ can freely diffuse between regions characterized by different illumination levels. The illumination of dark segments $\mathrm{B}, \mathrm{C}, \mathrm{D}$ and $\mathrm{E}$ is constant and equals to $\phi_{2}$. For such illumination the period of oscillations inside a segment is $T_{2}$. The illumination of the segment A depends on time. For $t \leq 100$ it is equal to $\phi_{2}$. For times $100 \leq t \leq 300$ the illumination of A segment decreases to $\phi_{1}$ and the period of oscillations in this segment becomes $T_{1}$. For $t \geq 300$ the illumination of the first dark segment increases again to $\phi_{2}$ and the period of oscillations increases to $T_{2}$. The dark segments are separated by illuminated gaps. For illumination level $\phi_{0}$ the considered reaction kinetics has a single, strongly attracting stationary state characterized by a low concentration of the activator $\left(u_{s}=0.0002\right)$.

If the illuminated gaps are wide ( 6 grid points, $\left.\Delta_{x}=0.05\right)$ then the interactions between dark segments are weak and oscillations are uncorrelated. The time evolution is fully determined by the initial condition. The increase of oscillation frequency in the segment A has not influence the other the time evolution in other segments.

If the illuminated gaps separating dark segments are narrow (5 grid points) then oscillations in segments correlate via diffusion of activator. In 
the case illustrated in Fig. 2b high frequency oscillations in A segment force fast oscillations in the segment B for $t \geq 100$. However, it the concentration of activator is just plotted as a function of time then it is quite difficult to spot the change in oscillation frequency, sepecially if the presented result extends over many cycles (cf. Fig. 2b). In order to distinguish and visualize slow and fast oscillations at a given point of medium we consider a function $D$ that maps time $t$ on the difference between the moment of the next maximum of activator concentration at the selected point and the current time:

$$
D(t)=\gamma(t)-t
$$

where, $\gamma(t)$ is the time when the next maximum of activator concentration after time $t$ appears. For an oscillating medium $D(t)$ plotted in the linear $x$ and $y$ - axis is a triangular function. $D(t)=0$ at the moments when excitation reaches its peak. The maximum of $D(t)$ equals to the oscillation period. Fig. 2c shows that TRUE and FALSE states, defined for the considered reaction, can be more precisely distinguished on a plot of $D(t)$ than on a plot presenting activator concentration as a function of time (Fig. 2b). The differences can even better visualized if the values of $D(t)$ are transformed using a nonlinear function that enhances differences between $T_{1}$ and $T_{2}$. Here we use a nonlinear transformation:

$$
t \rightarrow \tau(t)=\frac{1}{1+\exp \left(-\alpha \cdot\left(t-T_{c}\right)\right)}
$$

with $\alpha=3$. The function $\tau(t)$ is shown in Fig 1b. Figure 1c illustrates that the relative differences between $\tau_{1}$ and $\tau_{2}\left(\tau_{i}=\tau\left(T_{i}\right)\right.$ for $\left.i=1,2\right)$ are significantly better pronounced than those between $T_{1}$ and $T_{2}$. Small values of $\tau(D(T))$ indicate high frequency of oscillations, like those with period $T_{1}$. 
Fig. 2d shows that shortly after the fast oscillation in segment A appear the high frequency oscillations are subsequently forced in the segments B, C, D and E. The expansion of high frequency oscillations leads to a propagation of forced oscillations in an excitable medium. This propagation proceeds with a constant velocity. On the other hand if illumination in segment A increases and the system is no longer perturbed with a high frequency pulses than the periods of oscillations increase almost instantaneously in all segments.

\subsection{Signal channel for frequency coding}

Simulations of one-dimensional photosensitive medium demonstrated that locally forced high frequency oscillations in a compartmented oscillatory medium behave similarly to spikes in a spatially distributed excitable medium introduced by a local, external stimulus. Like spikes, high frequency oscillations spread out in space and they can vanish when the generating stimulus disappears. In the following we show that the basic concepts of information processing with excitation pulses propagating in a compartmented medium can be translated onto oscillatory medium and information coded with the frequency of oscillations. Let us consider a medium composed of dark disks on illuminated background. The medium is very similar to that studied in $[17,18,19,20,21]$, but in these papers dark disks were sub-excitable, whereas here the medium inside dark disks is oscillatory. As an experimental realization of such medium one can use a membrane with immobilised catalyst of photosensitive BZ that is placed inside a solution of the other reagents [29]. An external illumination can be applied to define the structure of dark disks on illuminated background. The period of oscillations 
inside a disk is controlled by illumination level according to Fig. 1.

In a two-dimensional system the number of neighbours is larger than in one-dimensional case and so the connectivity of disks can be more complex. This feature obviously extends the information processing functionality. It is also well known that oscillation period increases when a disk gets smaller [26]. Within the considered reaction model the effect can be explained by diffusion of activator to the surrounding non-excitable region where it quickly degenerates. The activator outflow depends on surface-to-volume ratio, so size dependent changes in period are more pronounced for small droplets. For selected parameters of the model the period of oscillations as a function of disk radius is illustrated in Fig. 3. The critical radius below which a disk does not oscillate strongly depends on illumination level and for $\phi_{2}$ is about 3 times larger than for $\phi_{1}$. The dependence of self-oscillation period on disk radius gives us another parameter that can be used to control propagation of high frequency oscillations in a droplet array.

\subsection{A signal channel constructed with disks}

In the following subsections we present BZ-disk based realizations of a few basic units that can be used to construct more complex information processing devices. Let us start with a signal channel within which chemical signals can be transmitted. Fig. 4 illustrates propagation of high frequency oscillations through an array of 5 disks. The radius of each disk is 80 grid points. The illumination of medium between disks is $\phi_{0}$. The leftmost disk, characterized by a low illumination $\left(\phi_{1}\right)$ oscillates with a higher frequency than the others, illuminated with the light intensity $\phi_{2}$. The gap between disks 


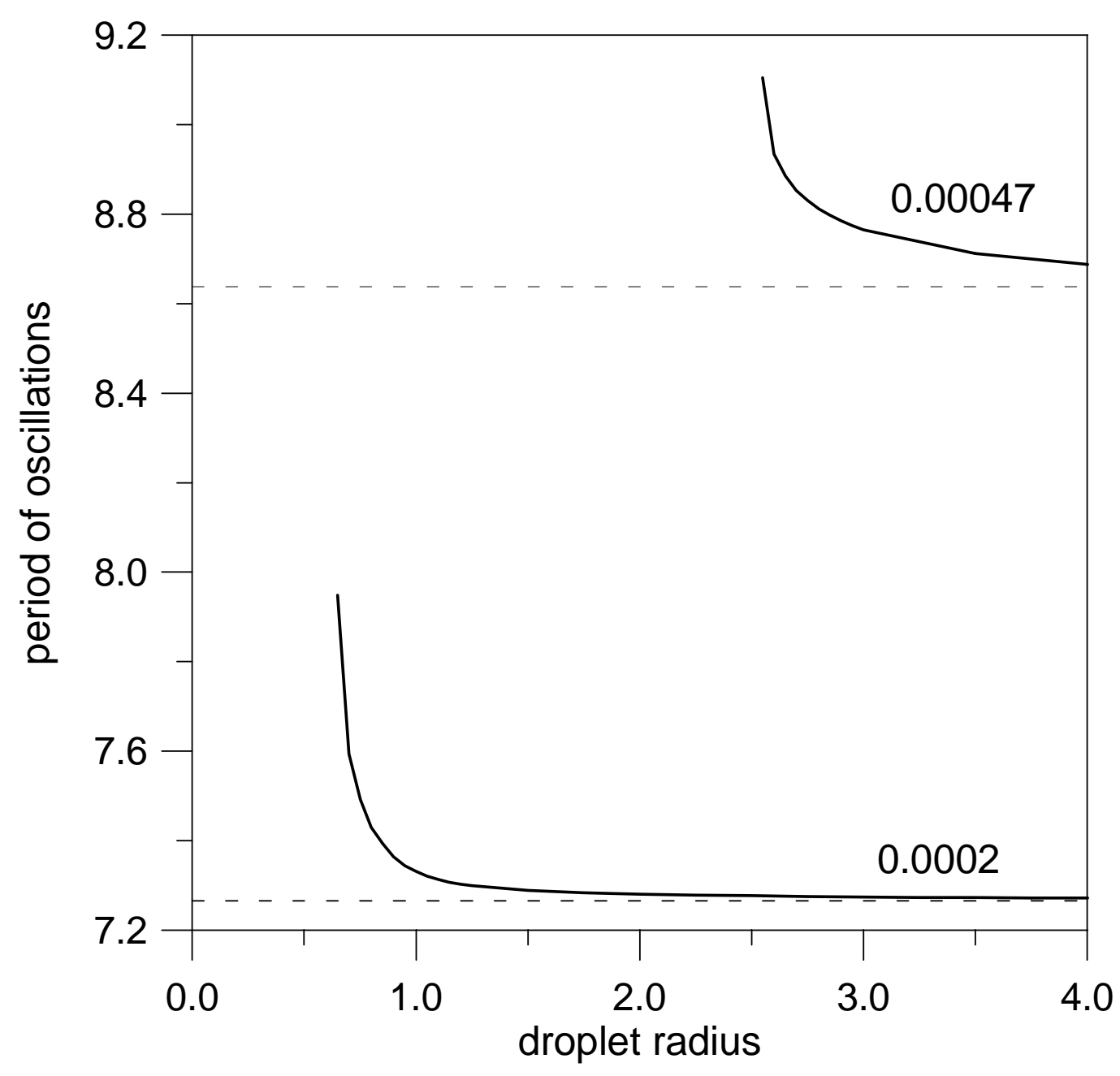

Figure 3: The period of oscillations in a dark disk as a function of its radius. A dark disk is surrounded by illuminated medium $\left(\phi_{0}=0.25\right)$. The results for two illuminations of a dark disk: $\phi_{1}=0.0002$ and $\phi_{2}=0.00047$ are shown. 
measured at the line of centers is 5 grid points. The time evolution of oscillations in coupled disks is illustrated in Figs. 4b and 4c that show $\tau(D(t))$ calculated at disk centers. As seen on Fig. 4b for a narrow non-excitable gap separating disks $\left(\Delta_{x}=\Delta_{y}=0.04\right.$, the gap is 0.20 distance unit wide ) the disk A oscillating with the highest frequency immediately dominates the time evolution of the whole array. The oscillations that start with initial random phases are synchronized within a single period. If the grid distances are larger $\Delta_{x}=\Delta_{y}=0.05$ (now the gap is 0.025 distance unit wide) then the coupling between disks is weaker and synchronization of oscillations takes around 40 time units (Fig.4c). For yet wider gaps the oscillations in disks do not synchronize. Fig. 4 shows that the array of disks placed close one to another can play the role of a signal channel for information coded in the frequency of oscillations.

\subsection{The OR gate}

High frequency oscillations can propagate through an array of coupled disks therefore two joined arrays should work as the OR gate for information coded in oscillation frequency. Let us consider a structure of disks illustrated on Fig. 5a. The leftmost disk (I1) and the rightmost disk (I2) are considered as the gate inputs and the top disk is the gate output $(\mathrm{O})$. The illumination of all disks except I1 and I2 is fixed and equal to $\phi_{2}$, so the periods of unforced oscillations on these disks are longer than the critical. The illumination of I1 and I2 can be $\phi_{1}$ or $\phi_{2}$ depending if the input represents TRUE or FALSE state. In numerical simulations of the time evolution we used a square grid with $\Delta_{x}=\Delta_{y}=0.045$. The radii of all disks are the same and equal to 60 
(a)
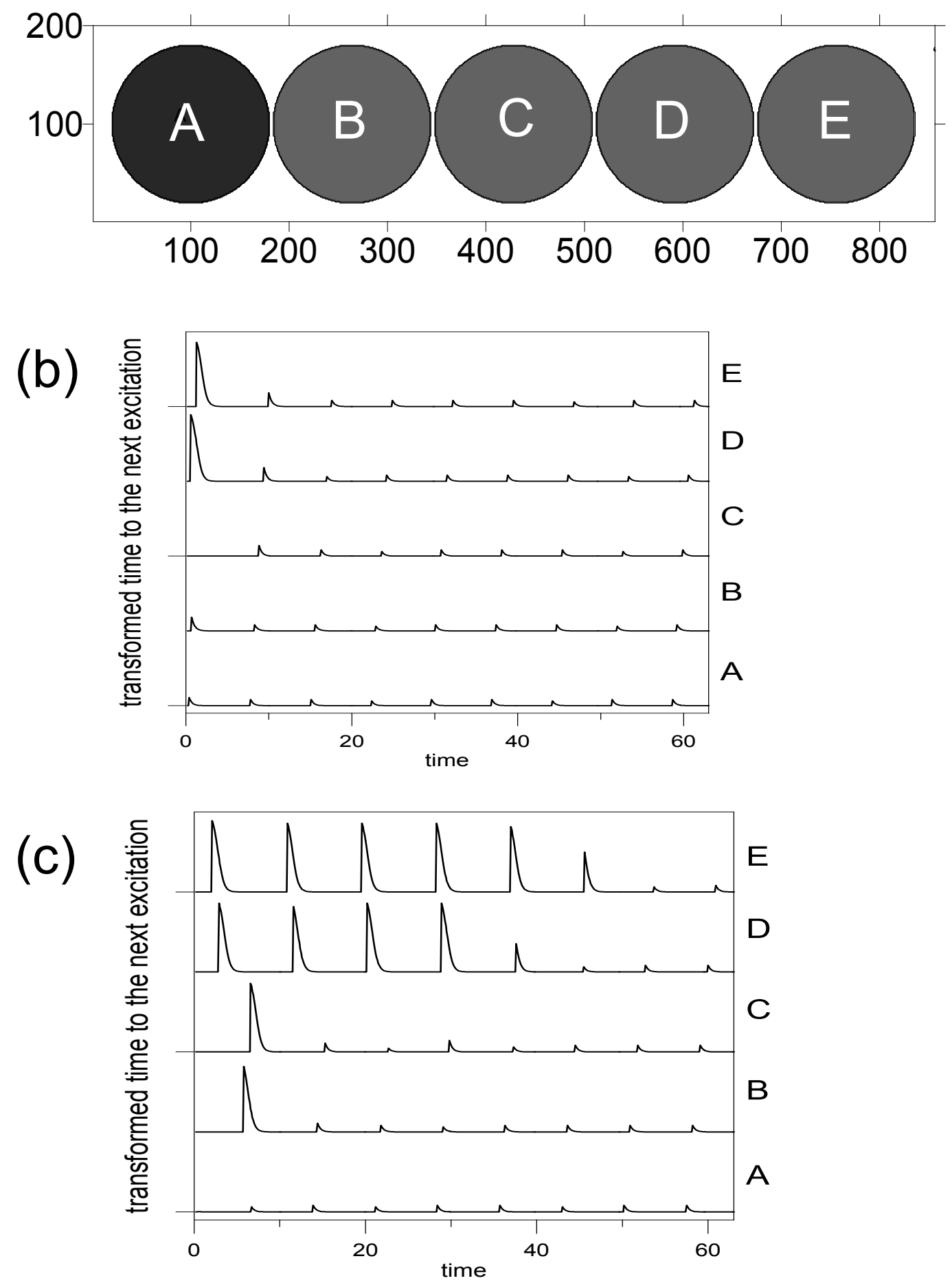

Figure 4: A signal channel formed by an array of disks. (a) A system's 17

geometry; illumination level is marked by grayscale: dark disk $\phi_{1}=0.0002$, gray disks $\phi_{2}=0.00047$, the white area around $\phi=0.25$. (b),(c) The function $\tau(D(t))$ calculated at disk centers for the following gaps separating disks: 0.20 distance units (b) and 0.25 distance units (c). 
grid points (2.7 distance units). The disks are separated by highly illuminated $\phi_{0}$ region and the widths of separating gaps at the line of centers are 5 grid points.

Fig 5b shows the transformed time to the next excitation when both inputs are in the FALSE state. In such case all disks synchronize in phase at the low frequency oscillations. The type of synchronization depends on the initial condition. The synchronization of oscillations means that even if initially the central disk $\mathrm{C}$ is frequently perturbed by different phase oscillations of its neighbors then after one or two cycles its oscillations synchronize with one of the neighbors and frequency of oscillations drops below $T_{c}$. Therefore, when both inputs are in the FALSE state the output state is also FALSE. Fig $5 \mathrm{c}$ shows the transformed time to the next excitation when the input disk I1 oscillates with a high frequency (its logic state is TRUE) and the input I2 oscillates with a low frequency, so its state is FALSE. Now fast oscillations of I1 expand over the whole system and the output represents TRUE state. Fig. 5d illustrates the case when both input disks I1 and I2 oscillate with a high frequency, so both inputs are in the TRUE state. Similarly to the case shown in Fig. 5c the output disk oscillates with a high frequency so its state is TRUE. Therefore the the disk structure illustrated on Fig. 5a operates as the OR gate.

\subsection{The negation gate}

The structure of disks that functions as the negation gate is illustrated in Fig. 6a. The time evolution of the system of disks has been studied on a square grid with $\Delta_{x}=\Delta_{y}=0.045$. The gate is composed of six disks 

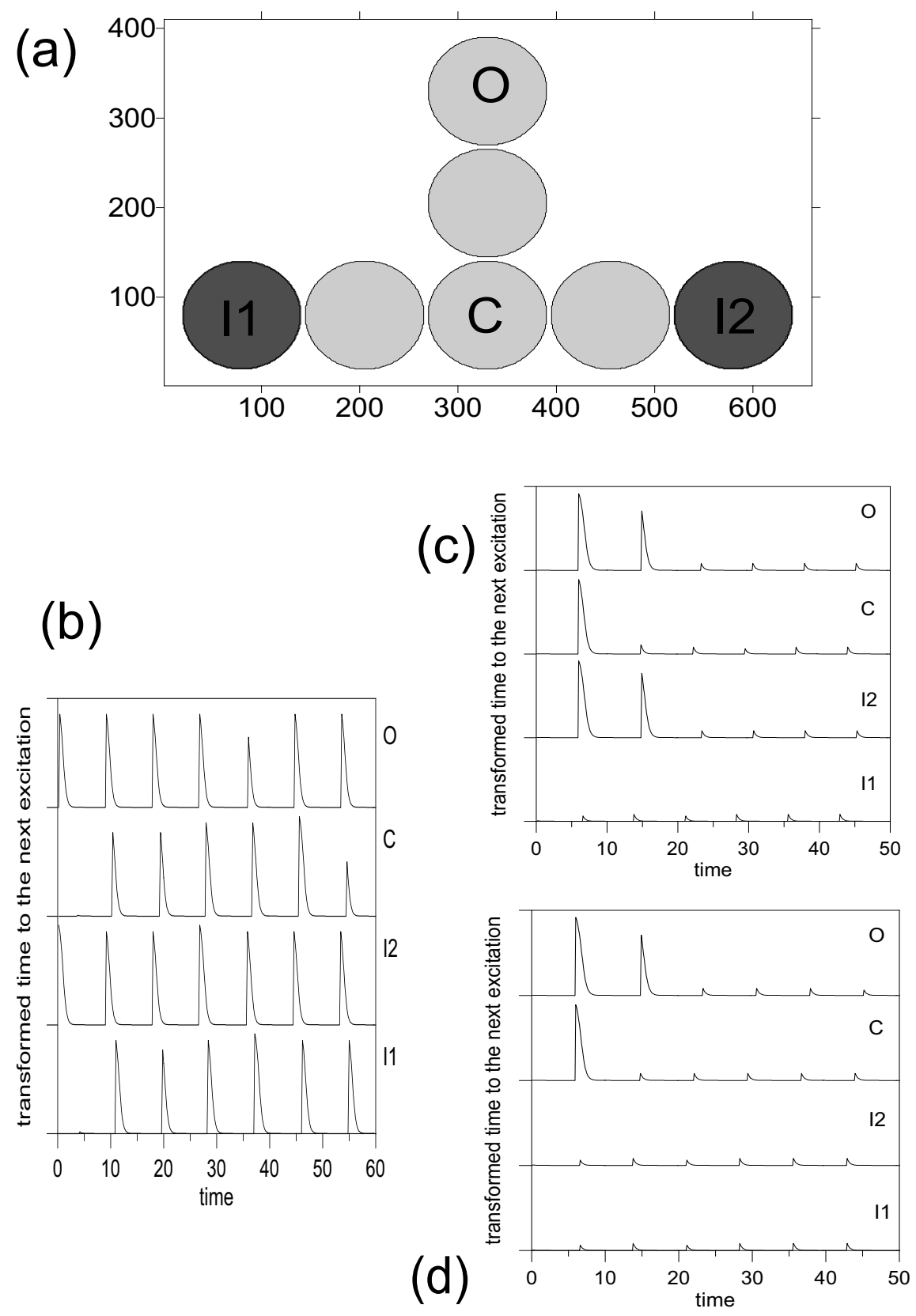

Figure 5: The OR gate build with disks. (a) A system's geometry; illumination level is marked by grayscale: dark input disk I1 and I2 can have illuminations $\phi_{1}=0.0002$ or $\phi_{2}=0.00047$ depending on the input, illuminations of grey disks is $\phi_{2}$, of the white 19 background $\phi_{0}$. (b,c,d) The function $\tau(D(t))$ calculated at centres of selected disks. (b) the state of both inputs is FALSE (illumination $\phi_{2}$ at I1 and I2). (c) The state of input I1 is TRUE (illumination $\phi_{1}$ at I1) and the state of input I2 is FALSE (illumination $\phi_{2}$ at I2)). (d) The state of both inputs is TRUE (illumination $\phi_{1}$ at I1 and I2). 
with different diameters separated by the highly illuminated medium $\left(\phi_{0}\right)$. The width of separating gaps measured at the line of disks centers is 5 grid points. The radius of the central small disk $\mathrm{C}$ is 28 grid points. Three disks that contact $\mathrm{C}$ have the same radii equal to 50 grid points (2.25 distance units). The illumination of disk $\mathrm{C}$ and its neighbors is fixed and equal to $\phi_{2}$. At such illumination level all these disks are too small to self-oscillate, but they can undergo excitations if they are externally perturbed by a periodic stimulus. The rightmost disk in the structure $(\mathrm{O})$ is the gate output. The radii of input disks I1 and I2 are equal to 60 grid points (2.7 distance units). The disk I2 plays the role gate input and its illumination can equal to $\phi_{1}$ (TRUE state at the input) or $\phi_{2}$ (input in the FALSE state). We assume that illumination of the disk I1 is fixed and equal to $\phi_{1}$ so it oscillates with period shorter than the critical. If the disk I2 is in the FALSE state then the evolution of the system is governed by high frequency oscillations of I1 disk. The numerical solution of reaction diffusion equations (cf. Fig. 6b) shows that the gate output exhibits complex oscillates $[27,28]$ with a high frequency. A complete oscillation cycle is composed of 5 excitation peaks and 4 of them are separated by time intervals shorter than $T_{c}$. Such oscillations represent the output in the TRUE state. On the other hand if the input I2 is in the TRUE state then oscillations at the output disk are periodic but with the period equal to $2 \cdot T_{2}$. In this case the output disk is in the FALSE state. Therefore the structure of disks shown in Fig. 6 functions as the NOT gate. 


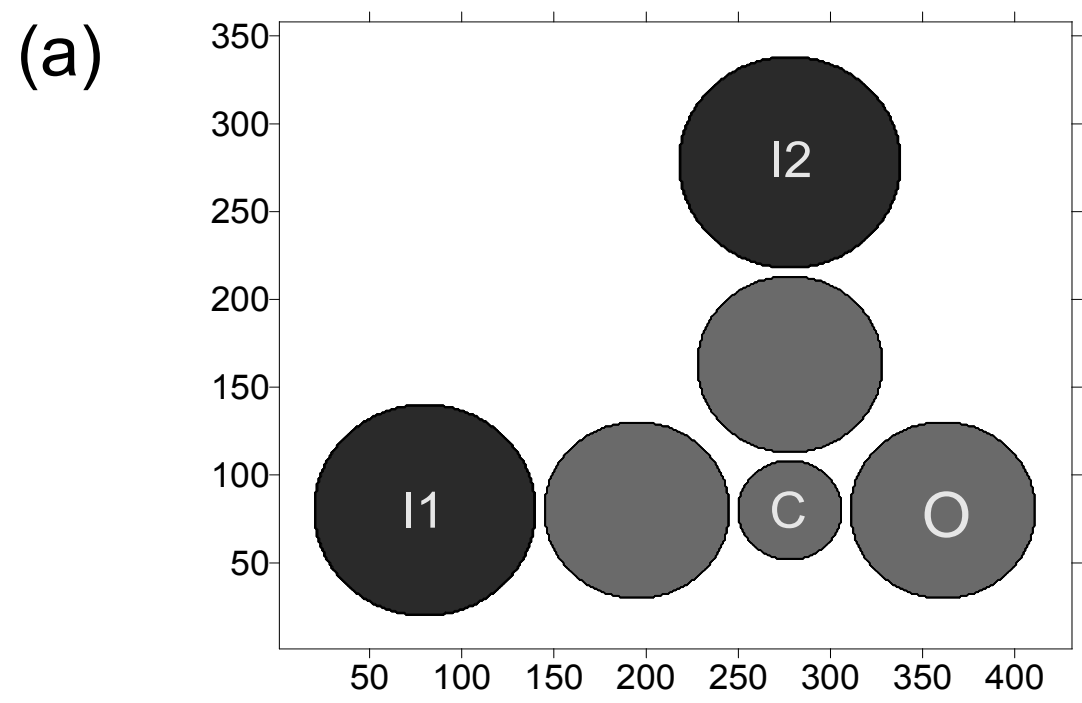

(b)

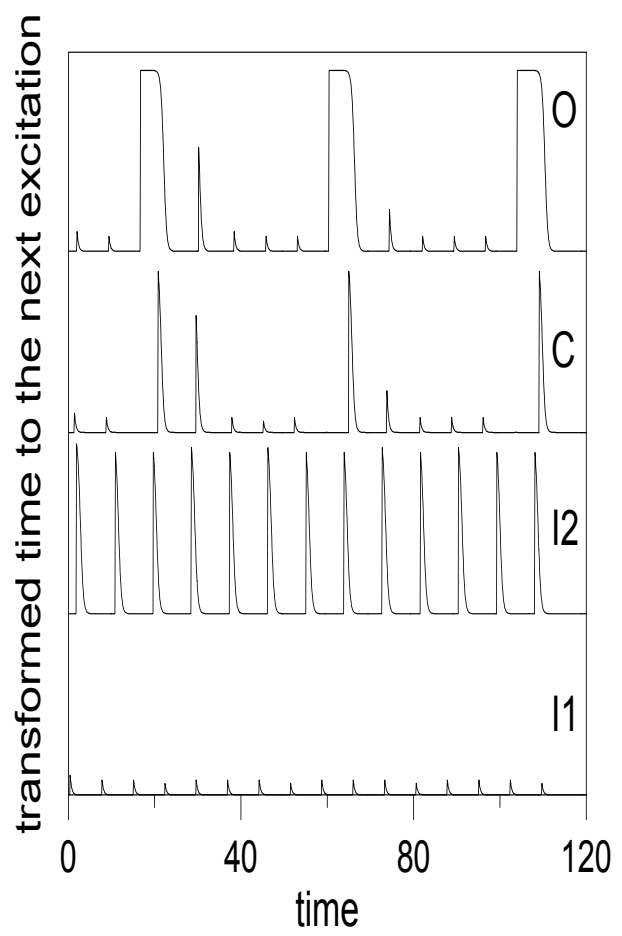

(c)

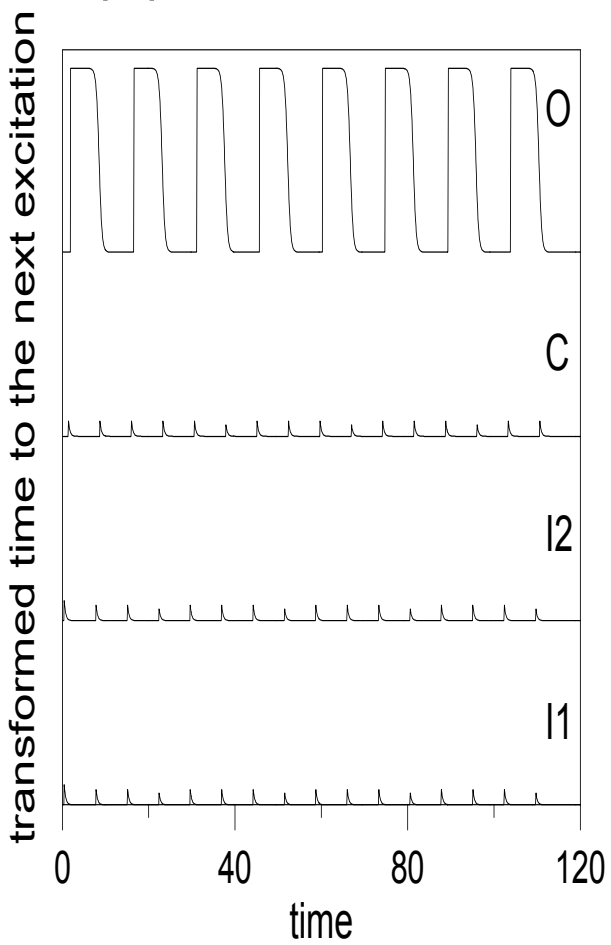

Figure 6: (a) The geometry of an array of disks that forms a NOT gate. (b, c) The function $\tau(D(t))$ calculated at centres of selected disks illustrated in Fig. 6a. (b) the input I2 is in the FALSE state. (c) The input I2 is in the TRUE state. 


\subsection{The signal diode}

A signal diode that transmits signals in one direction only is an important component of many information processing devices because it can be used to separate input channels form the region of medium where information is processed and thus eliminates potential interferences. If frequency of excitations is used for information coding than the signal diode is a device that transmits a high frequency stimulus in one direction and does not transmit it in the reverse direction. Having in mind a successful realization of a chemical signal diode with a junction between triangular and rectangular channels [9] we considered a chain of 5 disks with the radii equal to $70,70,140,20$ and 70 grid points respectively. The geometry of such disk array is illustrated in Fig. $7 \mathrm{a}$ and $7 \mathrm{c}$. The disks were separated by 5 grid points of the strongly illuminated medium (illumination $\phi_{0}$ ). The illumination level in all disks except the input one was $\phi_{2}$. Numerical simulations were performed on a square grid with $\Delta_{x}=\Delta_{y}=0.040$. The input signal was introduced from the input disk that oscillates with a high frequency because its illumination is low $\left(\phi_{1}\right)$. The disk at the other end of the chain represents diode output (O). The time evolution of oscillation in the array have been studied for both orientations of the big and the small disk with respect to the input disk. Figs. $7 \mathrm{~b}$ and $7 \mathrm{~d}$ show the transformed time between successive maxima of activator concentration at the centers of selected disks for a high frequency stimulus arriving from both directions. The output signals are different. If a train of high frequency pulses propagates from the big disk towards the small one we observe high frequency oscillations at the output disk. They are complex, composed of one long and one short period and the average time between excitations 
is shorter than $T_{c}$. For the reversed orientation of big and small disks with respect to the high frequency input (c.f. Figs. 7c and 7d) high frequency oscillations of a small disk B are too weak to force oscillations at the disk C. It oscillates with the period $T_{2}>T_{c}$ and synchronises with the output O. Therefore, the array of disks presented at Fig. 7 functions as a chemical signal diode for a signal coded in oscillation frequency.

\subsection{One bit memory}

If a medium is excitable than a ring-like structure can support stable rotation of an excitation pulse provided that the ring diameter is large enough [30]. For information coded in excitation pulses a ring that supports stable rotation can be used as a single bit memory cell. The presence of rotating excitation represents memory at the TRUE state and a ring in its stationary state corresponds to the logical FALSE [31, 32]. The idea of ring-shaped memory can be adopted to information coded in oscillation frequency. Let us consider a ring-like structure build of eight interacting disks, separated by a highly illuminated medium (illumination $\phi_{0}$ ) as illustrated in Fig.8a. The time evolution of disk oscillations has been studied on a square grid with $\Delta_{x}=\Delta_{y}=0.040$. All disks have the same diameter 80 grid points (3.2 distance units). Having in mind that forced high frequency oscillations spread out in an array of oscillating disks (cf. Fig. 4) we can initiate stable high frequency oscillations on the ring of oscillating disks in a way similar to initiation of rotating excitation on ring-shaped excitable medium. A frequency of chemical oscillations increases when we reduce disk illumination. A disk oscillating with a high frequency induces such oscillations on the neighbor- 
(a)

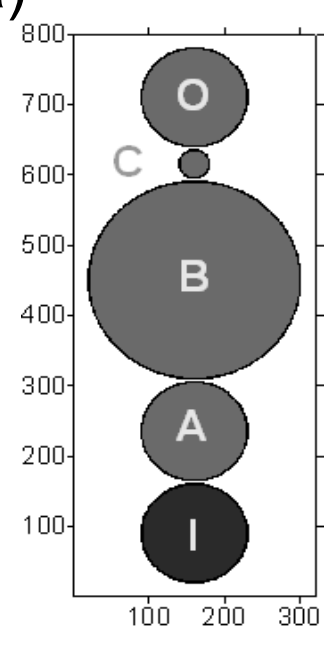

(c)

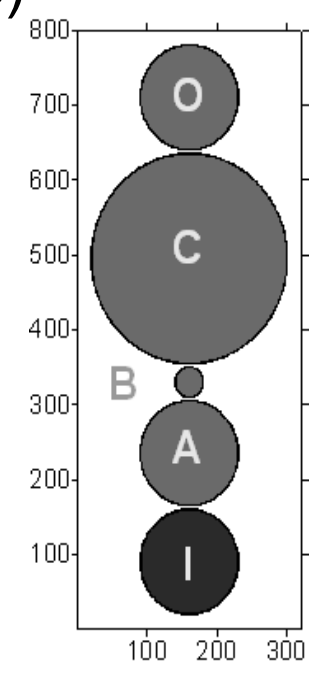

(b)

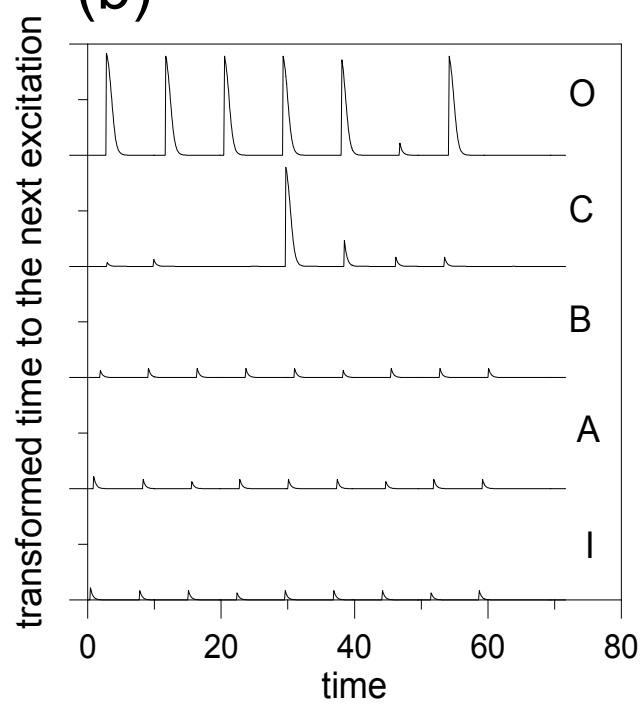

(d)

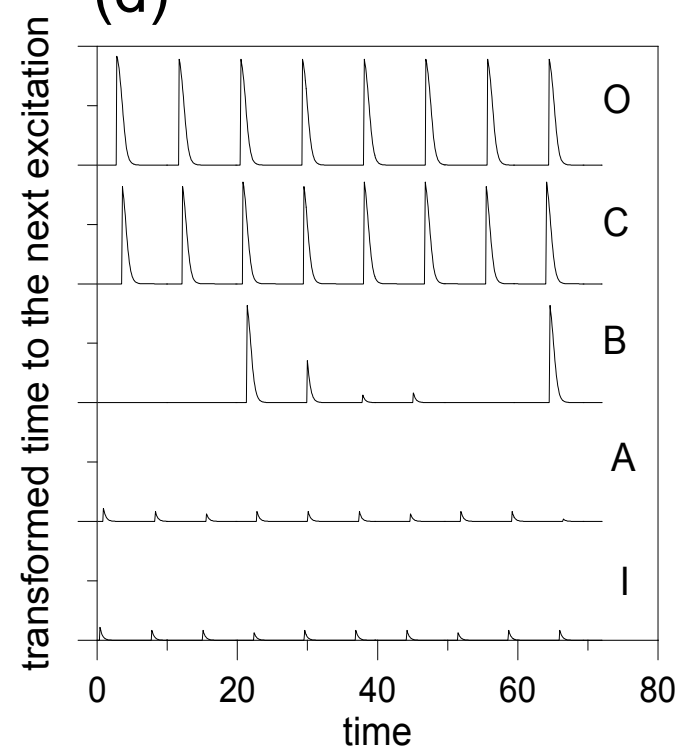

Figure 7: The geometry of an array of disks that forms a signal diode and the time evolution of activator at disk centers. The illumination level is marked by grayscale: dark input disk $\phi_{1}$, grey disk $\phi_{2}$, the white area around $\phi_{0}$. Figures on the right show the transformed time between successive maxima of activator concentration at the centers of selected disks for a high frequency stimulus arriving from the direction indicated on the left figure. 
ing disks so, the forced oscillations originating from a single disk spread out clockwise and anticlockwise round the ring. One of those directions should be blocked in order to get unidirectional rotation of forced high frequency oscillations. This can be done by unsymmetrical (with respect to the source) illumination of one disk. Alternatively one can use a signal diode inserted somewhere on the ring [32], but it makes the system more complex and in the following we discuss the first scenario.

Figure $8 \mathrm{~b}$ shows illuminations off all disks as a function of time. The illumination of disks B, C, E, G and $\mathrm{H}$ is constant and equal to $\phi_{2}=0.00047$ so periods of oscillations at these disks is $T_{2}$. High frequency oscillations is introduced by the disk $\mathrm{A}$. At the beginning its illumination was $\phi_{2}$, but during the time interval $t \in[20,30]$ it drops to $\phi_{1}$. Within this time interval oscillations at the disk $\mathrm{A}$ were faster than those of its neighbors and the disk A perturbed them with high frequency excitations. The stimulus was switched off for $t>30$ and illumination of disk A returned to the initial value $\phi_{2}$. In order to force unidirectional rotation of high frequency oscillations the symmetry of expansion was broken at the disk $\mathrm{F}$ where a high illumination level $\phi_{0}$ was applied for $t \in[20,30]$. At such illumination level this disk does not oscillate, but still the stimulus arriving from the disk E can excite the disk F. After such time dependent illumination a rotating excitation appears on the ring. The period of forced oscillations is short and equals to 5.2 time units. The period depends on two factors. Its lower boundary is determined by the refractory phase of chemical oscillations within which the medium does not answer to external perturbations. If the time I long enough to reexcite a disk then period of forced oscillations is given by the time within 
which an excitation rotates on the ring. It depends on the number of disks and on disk diameters. Using a fev more disks one can make it equal to $T_{1}$. Of course if there are too many disks and the time of rotation is longer than period of disk oscillations then rotating pulse becomes unstable and vanishes. The memory can be erased by breaking the rotating excitation by making one of the disks non-excitable. In our simulation we did it by illuminating an arbitrary disk (here the disk D) with $\phi_{0}$ for a time similar to the oscillation period (here 5 time units, see long dashed line on Fig. 8b).

\section{Discussion}

The majority of studies on chemical computing with reaction-diffusion processes is concerned with the Boolian information coding based on the presence of excitation pulses at selected points of the medium. It has been proven that such coding leads to required results. The chemical realization of many simple information processing devices based on specific geometrical distribution of excitable and non-excitable regions has been described $[7,33,34]$. However, in order to generate such devices a hand (and a head ) of an experimentalists is needed. It is more challenging to find a medium in which self-generation of information processing devices at carefully selected nonequilibrium conditions can occur. For example droplets containing reagents of BZ-reaction seems to be flexible enough to be self-assambled under the control of external stimuli, like for example a forced hydrodynamic flow in a cleverly selected geometry. For the simplest applications, where the number of connections between objects can be restriced to a few, 2-D disks seem to be as good 3-D droplets. It can be shown that the basic informa- 
(a)
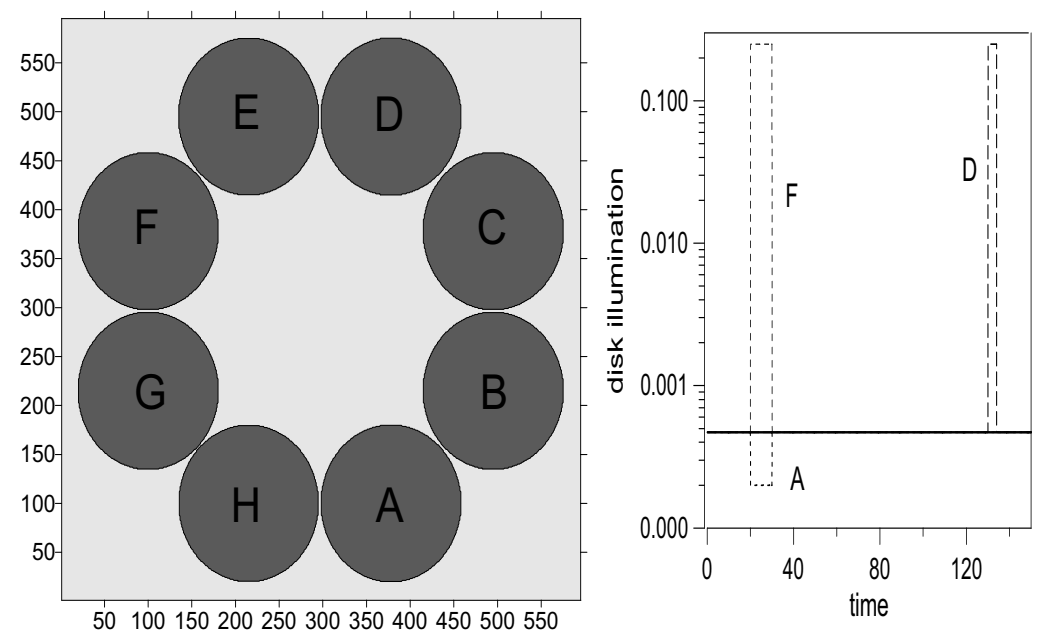

(c)

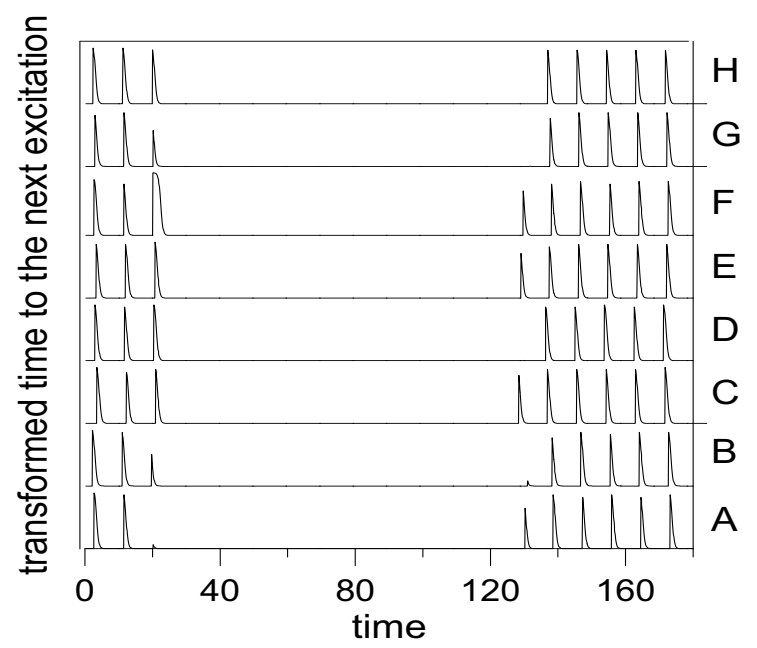

Figure 8: The geometry of a ring of disks that forms a memory cell. All disks have the same diameter 80 grid points and are separated by illuminated layer 5 grid points wide. , the white area around $\phi=0.25$. (b) The illumination of selected disks as a function of time: the solid line is an illumination of disks $\mathrm{B}, \mathrm{C}, \mathrm{E}, \mathrm{G}$ and $\mathrm{H}$, the short dashed line is illumination of the disk A, the medium dashed line is illumination of the disk F, the long dashed line is illumination of the disk D. (c) The function $\tau(D(t))$ calculated at centres of disks. 
tion processing devices can be constructed with excitable disks with precisely controlled excitability level and coding based on the presence of excitation pulses $[17,18,19,20,21]$. However, the recent experiments performed within the NEUNEU project [24] have shown that it is relatively easy to prepare a structure composed of interacting droplets containing oscillatory BZ medium separated by a non-oscillatory oil phase [23]. The lipid covered droplets are stable. They communicate via diffusion of activator trough the lipid bilayer and the oil phase. The droplet with the highest frequency of oscillations dominates the evolution of all droplets it is linked with. On the other hand it is quite difficult to make excitable BZ-droplets with controlled excitability level and force required excitations of selected number of them. These experimental findings encouraged us to present frequency based information coding that can be directly applied to a medium composed of oscillating droplets.

In this paper we demonstrated that the ideas of information processing devices described in [19] for excitable medium with information coding in the presence of excitation pulses translate directly onto an oscillatory medium with the frequency based information coding. Our arguments are based on numerical simulations with the same Oregonator model of a photosensitive $\mathrm{BZ}$ reaction that has been used in $[17,18,19]$. We have presented constructions of the basic logic gates (OR and negation) that make a functionally complete set of logical gates. Moreover we have shown that both one bit memory and a signal diode can be formed using a few disks. The agreement between simulations and experiments presented in $[17,18,19]$ was very good so we think that the presented gates, the diode and memory can be easily verified in experiments with a photosensitive BZ reaction and Ru-catalyst 
immobilized on a membrane. On the other hand simple, realistic models of kinetics of BZ droplets [25] are formally similar to Oregonator so a structure of droplets that performs a given operation should be similar to the presented structure of disks.

The numerical simulations indicate that construction of a disk system that performs a given function can be more stable for information coding with oscillation frequency than for information coding with the presence excitation pulses. We found that it is relatively easy to find values of parameters (for example radii of disks, space between disks or illumination level) for which a system of disks performs a given information processing operation if signal is frequency coded. In this paper the illumination levels corresponding to different Boolian states were quite randomly selected from the oscillatory domain. Contrary, a similar device processing information coded in the presence of excitation pulses requires much higher precision of conditions it operates at. For example, illumination levels at devices described in [35] should be known with 4-digit precision. Therefore, it should be easier for an experimentalist to build a device operating with information coded in frequency of chemical oscillations.

\section{Acknowledgements}

This work was supported by the NEUNEU project sponsored by the European Community within FP7-ICT-2009-4 ICT-4-8.3 - FET Proactive 3: Biochemistry-based Information Technology (CHEM-IT) program (project No. 248992). 


\section{References}

[1] Unconventional Computing 2007, edited by A. Adamatzky, B. De Lacy Costello, L. Bull, S. Stepney, and C. Teuscher (Luniver, Bristol, England, 2006).

[2] A. Adamatzky, B. De Lacy Costello, and T. Asai, Reaction- Diffusion Computers (Elsevier, New York, 2005).

[3] Unconventional Computing 2007, Eds.: A. Adamatzky, B. De Lacy Costello, L. Bull, S. Stepney, and C. Teuscher (Luniver, Bristol, England, 2006).

[4] Natural Computing, Eds.: Y. Suzuki, M. Hagiya, H. Umeo, A. Adamatzky (Springer, Tokyo, 2009).

[5] John von Neumann: Selected Letters, edited by M. Redei (American Mathematical Society, Providence, 2005).

[6] K. Yoshikawa, I.N. Motoike, T. Ichino, T. Yamaguchi, Y. Igarashi,J. Gorecki, J.N.Gorecka, Int J Unconv Computing 5, 3-37 (2009).

[7] J. Gorecki and J. N. Gorecka, in Encyclopedia of Complexity and Systems Science, edited by R. A. Meyers (Springer, Berlin, 2009).

[8] I. N. Motoike and K. Yoshikawa, Phys. Rev. E 59, 5354 (1999).

[9] T. Kusumi, T. Yamaguchi, R. R. Aliev, T. Amemiya, T. Ohmori, H. Hashimoto and K. Yoshikawa, Chem. Phys. Letters 271, 355 (1997).

[10] J. Gorecka and J. Gorecki, Phys Rev E 67, 067203 ( 2003). 
[11] A. Turing, Proc. London Math. Soc. 42, 230 (1936).

[12] A. Toth and K. Showalter, J. Chem. Phys. 103, 2058 (1995).

[13] O. Steinbock, A. Toth, and K. Showalter, Science 267, 868 (1995); O. Steinbock, P. Kettunen and K. Showalter, J. Phys. Chem. 100, 18970 (1996).

[14] J. Sielewiesiuk and J. Gorecki, J. Phys. Chem. A 105, 8189 (2001).

[15] A. Adamatzky and B. De Lacy Costello, Phys. Rev. E 66, 046112 (2002).

[16] A. Adamatzky, Chaos Solitons Fractals 21, 1259 (2004).

[17] A. Adamatzky, B. De Lacy Costello, L. Bull, and J. Holley, Isr. J. Chem. 51, $1(2011)$.

[18] A. Adamatzky, B. De Lacy Costello, and L. Bull, Int. J. Bifurcation Chaos 21, 1977 (2011).

[19] A. Adamatzky, J. Holley, L. Bull, and B. De Lacy Costello, Chaos Solitons Fractals 44, 779 (2011).

[20] J. Holley, A. Adamatzky, L. Bull, B. De Lacy Costello, and I. Jahan, Nano Commun. Networks 2, 50 (2011)

[21] J. Holley, Ishrat Jahan, B. De Lacy Costello, L. Bull, and A. Adamatzky, Phys. Rev. E 84, 056110 (2011).

[22] L. Kuhnert, Nature (London) 319, 393 (1986).

[23] J. Szymanski, J.N. Gorecka, Y. Igarashi: Int J Unconv Computing 7, 185-200 (2011). 
[24] NeuNeu Project, ArtificialWet Neuronal Networks from Compartmentalised Excitable Chemical Media, [http://neu-n.eu.]

[25] J. Gorecki, J. Szymanski, J.N. Gorecka, J. Phys. Chem. A 115, 88558859 (2011).

[26] O. Steinbock and S. C. Mu1ller, J. Phys. Chem. A 1998, 102, 6485-6490 ; K. Yoshikawa, R. Aihara and K. Agladze, J. Phys. Chem. A 1998, 102, 7649-7652.

[27] J. Sielewiesiuk and J. Gorecki, Phys. Rev. E 66, 016212 (2002).

[28] J. Sielewiesiuk and J. Gorecki, J. Phys. Chem. A 106, 4068 (2002).

[29] Jan Szymanski and J. Gorecki, Int J Unconv Computing 7, 50-60 (2011).

[30] A. Lazar, Z. Noszticzius, H-D. Forsterling and Z. Nagy-Ungvrai, Physica D 84, $112(1995)$

[31] I. N. Motoike, K. Yoshikawa, Y. Iguchi and S. Nakata, Phys. Rev. E 63, 036220 (2001).

[32] J. Gorecki and J. N. Gorecka On mathematical description of information processing in chemical systems, GAKUTO International, Mathematical Series and Applications 23, T. Aiki, N. Kenmochi, M. Niezgodka, M. Otani (Eds.), 73-90 (2005/2006).

[33] Guo-Mao Zhang, Ieong Wong, Meng-Ta Chou, and Xin Zhao, J. Chem. Phys. 136, 164108 (2012).

[34] Ming-Zhu Sun and Xin Zhao, J. Chem. Phys. 138, 114106 (2013). 
[35] Ben De Lacy Costello, A. Adamatzky, I. Jahan, Liang Zhang, Chemical Physics 381, 8899 (2011). 